\title{
Lipidomic analysis of Arabidopsis seed genetically engineered to contain DHA
}

\section{Xue-Rong Zhou ${ }^{1,2 *}$, Damien L. Callahan ${ }^{3,4}$, Pushkar Shrestha ${ }^{1}$, Qing Liu ${ }^{1}$, James R. Petrie ${ }^{1}$ and Surinder $P$. Singh ${ }^{1}$}

${ }^{1}$ Food Futures National Research Flagship, Commonwealth Scientific and Industrial Research Organisation, Canberra, ACT, Australia

2 Plant Industry, Commonwealth Scientific and Industrial Research Organisation, Canberra, ACT, Australia

${ }^{3}$ Metabolomics Australia, School of Botany, University of Melbourne, Melbourne, VIC, Australia

${ }^{4}$ Centre for Chemistry and Biotechnology, School of Life and Environmental Sciences, Deakin University, Melbourne, VIC, Australia

\section{Edited by:}

Xuemin Wang, University of

Missouri-St Louis \& Donald Danforth

Plant Science Center, USA

\section{Reviewed by:}

Philip David Bates, The University of

Southern Mississippi, USA

Chaofu Lu, Montana State

University, USA

\section{${ }^{*}$ Correspondence:}

Xue-Rong Zhou, CSIRO Plant

Industry, PO Box 1600, Clunies

Ross Street, Canberra, ACT 2601,

Australia

e-mail:xue-rong.zhou@csiro.au
Metabolic engineering of omega-3 long-chain $\left(\geq C_{20}\right)$ polyunsaturated fatty acids $(\omega 3$ LC-PUFA) in oilseeds has been one of the key targets in recent years. By expressing a transgenic pathway for enhancing the synthesis of the $\omega 3$ LC-PUFA docosahexaenoic acid (DHA) from endogenous $\alpha$-linolenic acid (ALA), we obtained the production of fish oil-like proportions of DHA in Arabidopsis seed oil. Liquid chromatography-mass spectrometry (LC-MS) was used to characterize the triacylglycerol (TAG), diacylglycerol (DAG) and phospholipid (PL) lipid classes in the transgenic and wild type Arabidopsis seeds at both developing and mature stages. The analysis identified the appearance of several abundant DHA-containing phosphatidylcholine (PC), DAG and TAG molecular species in mature seeds. The relative abundances of PL, DAG, and TAG species showed a preferred combination of LC-PUFA with ALA in the transgenic seeds, where LC-PUFA were esterified in positions usually occupied by 20:1 $\omega 9$. Trace amounts of di-DHA PC and tri-DHA TAG were identified and confirmed by high resolution MS/MS. Studying the lipidome in transgenic seeds provided insights into where DHA accumulated and combined with other fatty acids of neutral and phospholipids from the developing and mature seeds.

Keywords: Lipidomics, metabolic engineering, w3 LC-PUFA, oilseed, LC-MS, triacylglycerol, Arabidopsis

\section{INTRODUCTION}

Metabolic engineering of omega-3 long-chain polyunsaturated fatty acids ( $\omega 3$ LC-PUFA) in oilseed crops has been attempted by several groups in recent years due to the plateauing in global fishery stocks and the rise in demand for $\omega 3$ LC-PUFA containing fish oils (Haslam et al., 2013). Fish oils which contain omega-3 LC-PUFA such as eicosapentaenoic acid (EPA, 20:5 23 )

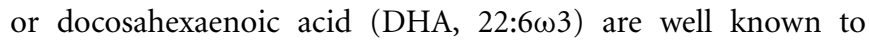
have anti-inflammatory properties although it is not known if EPA and/or DHA are involved in the suppression of cytokine production (James et al., 2000). The engineered DHA biosynthetic pathway in plant seeds involves multiple steps of desaturation and elongation of fatty acids in phosphatidylcholine (PC) and acyl-CoA pools. We had previously reported the first successful transgenic production of DHA in Arabidopsis seed in 2005 (Robert et al., 2005), although only at the level of $0.5 \%$ in seed triacylglycerol (TAG). This initial report was followed by the transgenic production of DHA in Brassica seed by $\mathrm{Wu}$ et al. (2005). Good progress has been made in engineering the $\omega 3$ LC-PUFA DHA since then (Petrie et al., 2012; Ruiz-Lopez et al., 2012, 2013b). Recently, we have shown that expressing seven genes for the DHA synthesis pathway in Arabidopsis with different seed specific promoters resulted in up to $15 \%$ DHA in seed oil (Petrie et al., 2012). This exceeded the $12 \%$ level at which DHA is generally found in bulk fish oil.

The DHA synthesis pathway consists of a series of fatty acid desaturation and elongation steps that are thought to occur in discrete seed lipid pools. Therefore, effects of transgenic expression of this pathway are expected to be expressed throughout the lipidome of the developing seed. Oil accumulation in the developing seed requires cross talk between the ER membrane lipids, predominantly represented by PC and other phospholipids, such as phosphatidic acid (PA), phosphatidylethanolamine (PE), phosphatidylglycerol (PG), phosphatidylinositol (PI) and phosphatidylserine (PS), soluble acyl CoA fatty acids and DAG lipid pools. The fatty acids in the acylCoA pool are then utilized by the acyl-CoA-dependent Kennedy pathway to be assembled into TAG. This involves the sequential acylation of glycerol-3-phosphate to produce phosphatidic acid (PA) by glycerol-3-phosphate acyltransferase (GPAT) and 1acyl-glycerol-3-phosphate acyltransferase (LPAAT), dephosphorylation of PA to diacylglycerol (DAG) by phosphatidic acid phosphatase (PAP), and the final acylation of DAG to TAG by diacylglycerol acyltransferase (DGAT) (Figure 1). In addition, de novo synthesized DAG can be used for synthesis of PC by a reversible cytidine- $5^{\prime}$-diphosphocholine:diacylglycerol cholinephosphotransferase (CPT) (Slack et al., 1983). However, 


FIGURE 1 I Simplified representation of lipid metabolism in plant cells.
Lipid pools are acyl-CoA, acyl co-enzyme A; LPC, Iysophosphatidylcholine;
PC, phosphatidylcholine; G-3-P, glycerol-3-phosphate; LPA, lysophosphatidic
acid; PA, phosphatidic acid; DAG, diacylglycerol; TAG, triacylglycerol. The
enzymes are LPCAT, lysophosphatidylcholine acyltransferase; PLD,
phospholipase D; CPT, CDP-choline:diacylglycerol
cholinephosphotransferase; PDCT, phosphatidylcholine:diacylglycerol
cholinephosphotransferase; PLC, phospholipase C; PDAT,
phospholipid:diacylglycerol acyltransferase; GPAT, glycerol-3-phosphate
acyltransferase; LPAAT, lysophosphatidic acid acyltransferase; PAP,
phosphatidic acid phosphatase; DGAT, diacylglycerol acyltransferase.

recent studies (Williams et al., 2000; Bates et al., 2012; Wang et al., 2012) have demonstrated that the acyl-editing cycle is the major pathway for PC synthesis. This cycle proceeds through the acylation of lysophosphatidylcholine (LPC) and deacylation of PC by acyl-CoA:lysophosphatidylcholine acyltransferase (LPCAT) (Stymne and Stobart, 1984; Bates et al., 2012; Wang et al., 2012). On the other hand, there is accumulating evidence of the use of PC-derived DAG for synthesis of PUFA-containing TAG in plants (Bates and Browse, 2012). The PC to DAG conversion is believed to be mainly carried out by phosphatidylcholine:diacylglycerol cholinephosphotransferase (PDCT) through phosphocholine head group exchange (Bates and Browse, 2012; Hu et al., 2012), although PC-derived DAG can also be synthesized by CPT, or phospholipase C.

As it is proposed that the fatty acyl chains in the PC and DAG pools direct the flux of engineered LC-PUFA products onto TAG, a detailed analysis of the lipids in TAG, DAG and PL in control and transgenic Arabidopsis seeds during seed development was required. Traditional gas chromatography (GC) analysis or thin layer chromatography (TLC) coupled with GC can provide an overall profile for each separate class. In the transgenic line GA7 expressing the entire DHA pathway, it was not clear how newly synthesized LC-PUFA's are directed toward the synthesis of neutral lipids, in particular their assembly in TAGs. Recently developed lipidomics techniques using liquid chromatographymass spectrometry (LC-MS) now provides a detailed composition of the majority of individual lipid molecular species and lipid classes present in cells and tissues (Han and Gross, 2005; Blanksby and Mitchell, 2010). This information will help elucidate the metabolic pathways and potential bottlenecks where the engineered fatty acids accumulate and combined with other endogenous fatty acids. The lipidomics analysis techniques have been successfully used in many applications (Watson, 2006; Ferreri and Chatgilialoglu, 2012; Maatta et al., 2012; Hyötyläinen et al., 2013). In this study we utilized the different scan functions of a triple
quadruple-MS (QQQ-MS), such as neutral loss, or precursor ion scan and targeted multiple reaction monitoring (MRM) to characterize the lipid profiles. A quadruple time of flight-MS (QTOF-MS) was also used to confirm the identities of some important lipid species.

\section{MATERIALS AND METHODS PLANT MATERIALS}

Arabidopsis thaliana Columbia (Col-0) wild type (WT) and DHA producing transgenic line GA7 (Petrie et al., 2012) were grown in a glasshouse at $22^{\circ} \mathrm{C}$ and a $16 / 8 \mathrm{~h}$ light and dark cycle. GA7 was generated by expressing the Lachancea kluyveri $\Delta 12$-desaturase, Pichia pastoris $\Delta 15$-/ $/ 33$-desaturase, Micromonas pusilla $\Delta 6$-desaturase, Pyramimonas cordata $\Delta 6$ - and $\Delta 5$ elongases and Pavlova salina $\Delta 5$ - and $\Delta 4$-desaturases. It has been shown previously that expression of these genes results in the accumulation of up to $15.1 \%$ DHA in total seed oil (Petrie et al., 2012). Developing seeds were harvested on dry ice at 10-12 days after flowering (DAF), frozen with liquid nitrogen and freeze dried prior to lipid analysis.

\section{TOTAL LIPID EXTRACTION}

Total lipids were extracted using methanol/chloroform from triplicate samples of the developing and the mature seeds (Zhou et al., 2013). Tri-17:0-TAG (Nuchek Prep, Elysian, MN, USA) was added based on the dry weight of developing or mature seeds, as an internal standard. Aliquots of the total lipid extracts from $1 \mathrm{mg}$ of seeds (dry weight) were dried under $\mathrm{N}_{2}$ followed by re-dissolving in $1 \mathrm{~mL}$ of butanol:methanol $(1: 1, \mathrm{v} / \mathrm{v})$ containing $10 \mathrm{mM}$ butylated hydroxytoluene prior to LC-MS analysis. The concentration of the lipids were measured and the relative amounts based on the same dry weight of seed were compared.

\section{TWO-DIMENSIONAL TLC}

The two-dimensional TLC analysis was carried out in one of the triplicate samples. Individual chloroplastidic and extra-chloroplastidic polar lipid classes were separated from the total lipids using two-dimensional TLC using chloroform:methanol:water $(65: 25: 4, \mathrm{v} / \mathrm{v} / \mathrm{v})$ as the first solvent system and chloroform:methanol: $\mathrm{NH}_{4} \mathrm{OH}$ :ethylpropylamine (65: $35: 5: 0.5, \mathrm{v} / \mathrm{v} / \mathrm{v} / \mathrm{v})$ as the second solvent system as described (Khozin et al., 1997). The lipid spots were visualized under UV after spraying $0.001 \%$ primuline in acetone/water $(4: 1, \mathrm{v} / \mathrm{v})$ and were collected for GC analysis with a known amount of 17:0 free fatty acid as internal standard as described previously (Zhou et al., 2013).

\section{LIPID PROFILING WITH LC-MS}

The extracted total lipids were analyzed using an Agilent 1200 series LC coupled to an Agilent 6410B electrospray ionization QQQ-MS (Agilent, Palo Alto, California, USA). A $5 \mu \mathrm{L}$ injection of each total lipid extract was chromatographically separated with an Ascentis Express RP-Amide $50 \times 2.1 \mathrm{~mm}$, $2.7 \mu \mathrm{m}$ HPLC column (Sigma-Aldrich, Castle Hill, Australia) using a binary gradient with a flow rate of $0.2 \mathrm{~mL} / \mathrm{min}$. The mobile phases were: A. $10 \mathrm{mM}$ ammonium formate in $\mathrm{H}_{2} \mathrm{O}$ :methanol:tetrahydrofuran (50:20:30, v/v/v.); B. $10 \mathrm{mM}$ 
ammonium formate in $\mathrm{H}_{2} \mathrm{O}$ :methanol:tetrahydrofuran (5:20:75, $\mathrm{v} / \mathrm{v} / \mathrm{v}$.). Selected neutral lipids (TAG and DAG) and phospholipids (PL, including PC, PE, PI, PS, PA, PG) were analyzed by multiple reaction monitoring (MRM) using a collision energy of $30 \mathrm{~V}$ and fragmentation energy of $60 \mathrm{~V}$. Neutral lipids were targeted on the following major fatty acids: 16:0 (palmitic acid), 18:0 (stearic

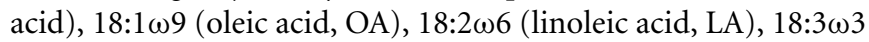

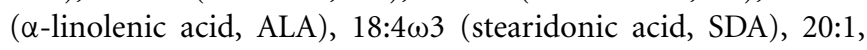
20:2, 20:3, 20:4 $\omega 3,20: 5 \omega 3,22: 4 \omega 3,22: 5 \omega 3,22: 6 \omega 3$, while phospholipids were scanned containing $\mathrm{C}_{16}, \mathrm{C}_{18}, \mathrm{C}_{20}$, and $\mathrm{C}_{22}$ species with double bonds of $0-3,0-4,0-5,4-6$ respectively. Individual MRMs for each TAG was based on ammoniated precursor ion and product ion from neutral loss of 20:1, SDA, EPA, and DHA. TAG and DAG were quantified using the $50 \mu \mathrm{M}$ tristearin and distearin injected in the same batch as external standards. Phospholipids were quantified with $10 \mu \mathrm{M}$ of di-18:0-PC, di-17:0-PA, di-17:0PE, 17:0-17:1-PG, di-18:1-PI and di-17:0-PS external standards (Avanti Polar Lipids, Alabaster, Alabama, USA) injected in the same batch, and presented as $\mu \mathrm{M}$ in the total lipid extract. All the LC-MS data were presented as average of triplicate samples with standard deviation, calculated with Microsoft Excel. Selected TAG, DAG, and PL species were further confirmed by Agilent 6520 Q-TOF MS/MS using the same chromatographic conditions as just described.

\section{RESULTS AND DISCUSSION}

\section{MEMBRANE PHOSPHOLIPID CHARACTERIZATION: INCREASED PHOSPHOLIPIDS IN TRANSGENIC LINE}

We previously showed that expressing seven enzymes for DHA biosynthesis pathway in Arabidopsis resulted in the efficient accumulation of DHA (up to 15.1\%) in total seed lipids of transgenic line GA7 (Petrie et al., 2012). These enzymes included L. kluyveri $\Delta 12$-desaturase, $P$. pastoris $\Delta 15-/ \omega 3$-desaturase, $M$. pusilla $\Delta 6$ desaturase, $P$. cordata $\Delta 6$ - and $\Delta 5$-elongases and $P$. salina $\Delta 5$ and $\Delta 4$-desaturases. GA7 showed significantly increased ALA and a reduced proportion of $20: 1$ in total seed lipids. In addition, GA7 accumulated only very low amounts of $\mathrm{C}_{20}$ intermediates. In the present study, we have further characterized the PL, DAG, and TAG species in detail using LC-MS analysis in MRM mode. Comparative LC-MS analysis was carried out on the developing and mature seeds of GA7 and wild type (WT) control in order to compare the lipid species.

An overall increase in PL was observed in GA7 when compared to the wild type seeds from the plants grown at the same time. The profiles and the amounts of the different PL classes were first analyzed by two-dimensional TLC followed by fatty acid analysis by GC, as the indicative result. The most abundant PL species, PC, in the Arabidopsis seeds, significantly increased (40\%) in GA7 mature seeds compared to WT mature seeds as shown in Table 1, based on seed dry weight. Detailed lipidomic profiling was then carried out using LC-MS on triplicate samples. The LC-MS data showed even higher than $40 \%$ increase of PC from WT to GA7 (see below). It has been shown in multiple plants that fatty acid engineering can reduce total TAG production and thus reduce seed weight (Dauk et al., 2007; Li et al., 2012; Bates et al., 2014). The expression of DHA synthesis pathway in Columbia did reduce the mature seed weight from $2.05 \pm 0.13 \mathrm{mg}$

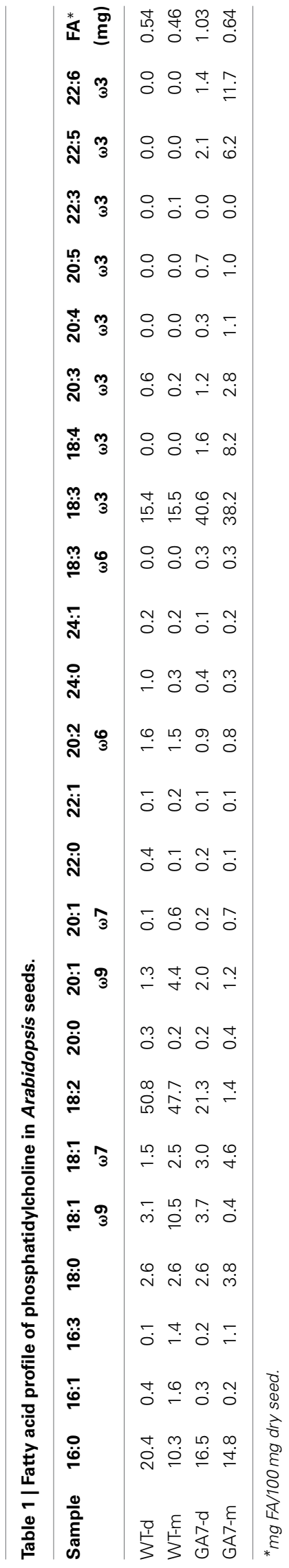


in WT to $1.89 \pm 0.03 \mathrm{mg}$ in GA7 per 100 seeds (five replicates). In other words, GA7 resulted in about $8 \%$ reduction in seed weight compared to WT. Although we did not analyse the lipids concentration per individual seed, it is clear that the $8 \%$ reduction of

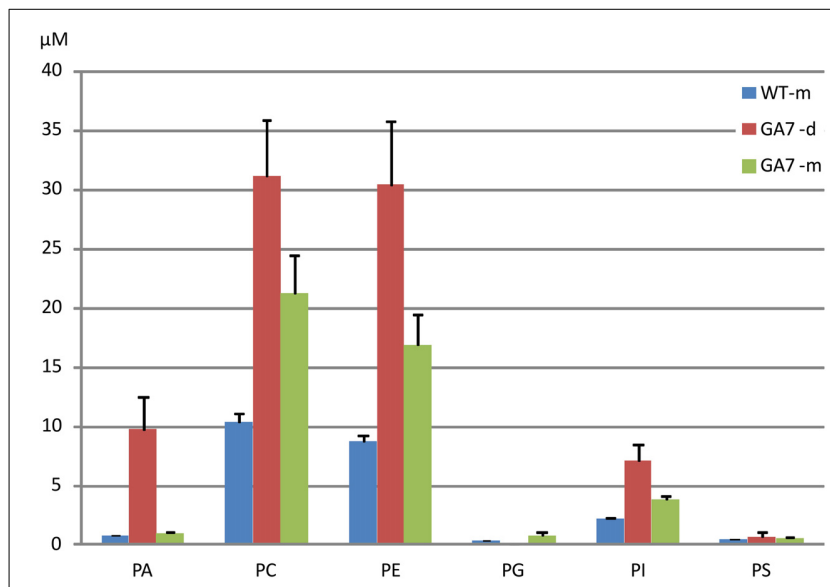

FIGURE 2 | Total phospholipids in developing and mature Arabidopsis seeds. Phosphatidic acid (PA), phosphatidylcholine (PC), phosphatidylethanolamine (PE), phosphatidylglycerol (PG), phosphatidylinositol (PI) and phosphatidylserine (PS) were determined from triplicate samples with the mean and standard deviation shown. WT-m, Columbia wild type mature seeds; GA7-d and GA7-m, developing and mature seeds of transgenic GA7 line containing a DHA biosynthetic pathway (genes are described in the text). Data are shown as the mean of triplicate analysis with the error bars representing the standard deviations, concentrations $(\mu \mathrm{M})$ have been determined in the extracts from the same amount of dry seed weight for both developing and mature seeds. seed weight compared with the $40 \%$ increase in PC from WT to GA7 still shows an increase in the levels of PC even on a per seed base. Both the WT and GA7 showed higher amounts of PC in the developing seeds (WT-d, GA7-d) than in their mature seeds (WT$\mathrm{m}$ and GA7-m). Further, GA7 had increased total amounts of PC in the developing (2.3-fold higher) and mature seeds (1.4-fold higher) than the WT mature seeds. Again, the amount of PC was expressed based on seed dry weight. Nevertheless, ectopic expression of five membrane-associated fatty acid desaturases resulted in an altered unsaturated fatty acid profile of the PC fraction in GA7. The levels of oleic acid and linoleic acid in PC showed a significant reduction, with decreased of 10.5-0.4 and 47.4-1.4\%, respectively. This is associated with a significant increase of newly synthesized $\omega 3$ and $\omega 6$ LC-PUFA. This result was confirmed by more detailed LC-MS analysis of PC species. Other PL classes followed a similar pattern of increase in GA7 compared to WT as described for PC, and altered the fatty acid profile similar to PC, i.e., a significant reduction of 18:2 and an associated increase of 18:3 and LC-PUFA based on seed dry weight (Table S1, and see detail LC-MS analysis below).

LC-MS analysis of the PL was focused on PA, PC, PE, PG, PI, and PS. The major fatty acids from palmitic acid to the end product $\omega 3$ DHA in the engineering pathway were analyzed. As shown in Figure 2, PC and PE were the major PL, followed by PA, with very low amounts of PG and PS. The LC-MS analysis confirmed that there were more total PLs in the developing seeds than in the mature seeds of GA7.

Both the developing and mature seeds of GA7 produced new LC-PUFA in phospholipids. PC precursor total ion scan showed that the WT seeds contained PC with total acyl chain lengths only up to $\mathrm{C}_{38}$ (PC 38:Y, in which a total of 38 carbons occurs

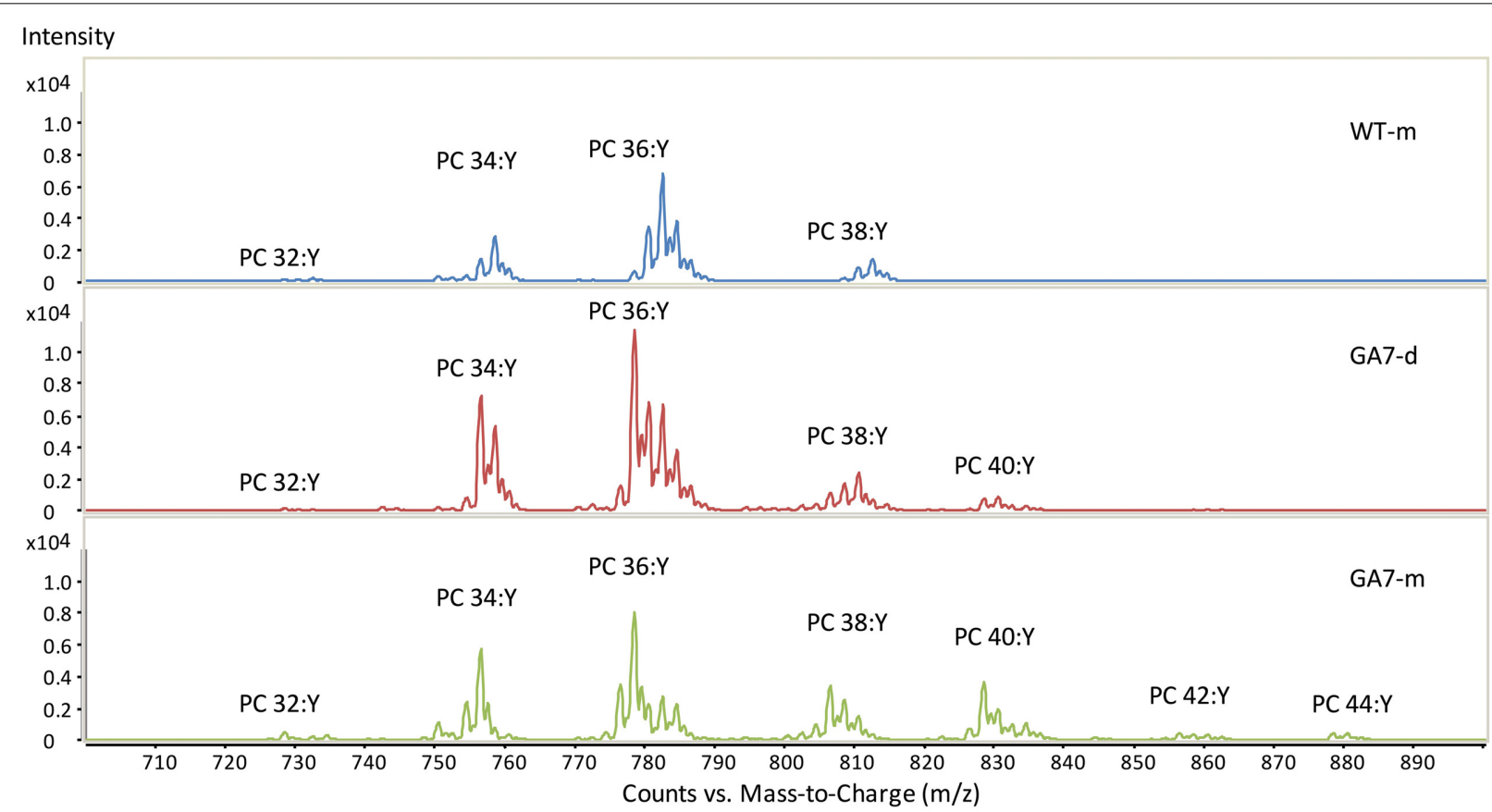

FIGURE 3 | Summed spectra from the precursor ion scan for phosphatidylcholine (PC) from the WT or GA7 Arabidopsis seeds. PC 36:Y indicates PC species with a total of 36 carbons with a total number of $Y$ double bonds in the two acyl chains. $Y$ axis represents the response of ion scan. 
in the two acyl chains on PC with a total double bond number of Y). The most likely fatty acid combination of being $\mathrm{C}_{18}$ and $\mathrm{C}_{20}$ (Figure 3). The WT only had low amounts of $\mathrm{C}_{20}$ fatty acids in PC (Table 1). The developing GA7 seeds produced PC 40:Y, indicating the esterification of two $\mathrm{C}_{20}$ fatty acids. In the mature GA7 seeds, there were low yet detectable amounts of $\mathrm{PC} 42: \mathrm{Y}$ and $\mathrm{PC} 44$ : $\mathrm{Y}$, i.e., $\mathrm{PC} \mathrm{C}_{20} / \mathrm{C}_{22}$ or $\mathrm{PC} \mathrm{C}_{22} / \mathrm{C}_{22}$, indicating the accumulation of LC-PUFA in the PC pool. Furthermore, precursor ion scan showed higher abundance of the earlier eluted part of the each PC chain length group in GA7 when compared to WT. The molecular species with more double bonds eluted slightly earlier than the molecular species with fewer double bonds in the group with same chain length. This indicated that the PC clusters in GA7 contain more double bonds. This was further supported by the quantification of the different PC species as shown in Figure 4A.

There was a large increase in PC $36: 6$ (PC 18:3/18:3 as the major species) in GA7 seeds, followed by PC 34:3 (PC 16:0/18:3 as the major species). PC species containing higher numbers of carbons and double bonds in their acyl chains such as PC 40:4 to PC 44:12 were only found in GA7, suggesting EPA or DHA also accumulated in the PC pool. There was a small amount of PC 44:12 $(0.9 \pm 0.01 \%$ out of total PC in mature GA7 seeds), which was likely PC 22:6/22:6. MS/MS analysis of this precursor $(878.6 \mathrm{~m} / \mathrm{z})$ by Q-TOF confirmed the loss of PC head group $(184.2 \mathrm{~m} / z)$ and the 22:6 fatty acid $(568.3 \mathrm{~m} / \mathrm{z})$, as shown in Figure $\mathbf{S 1}$.

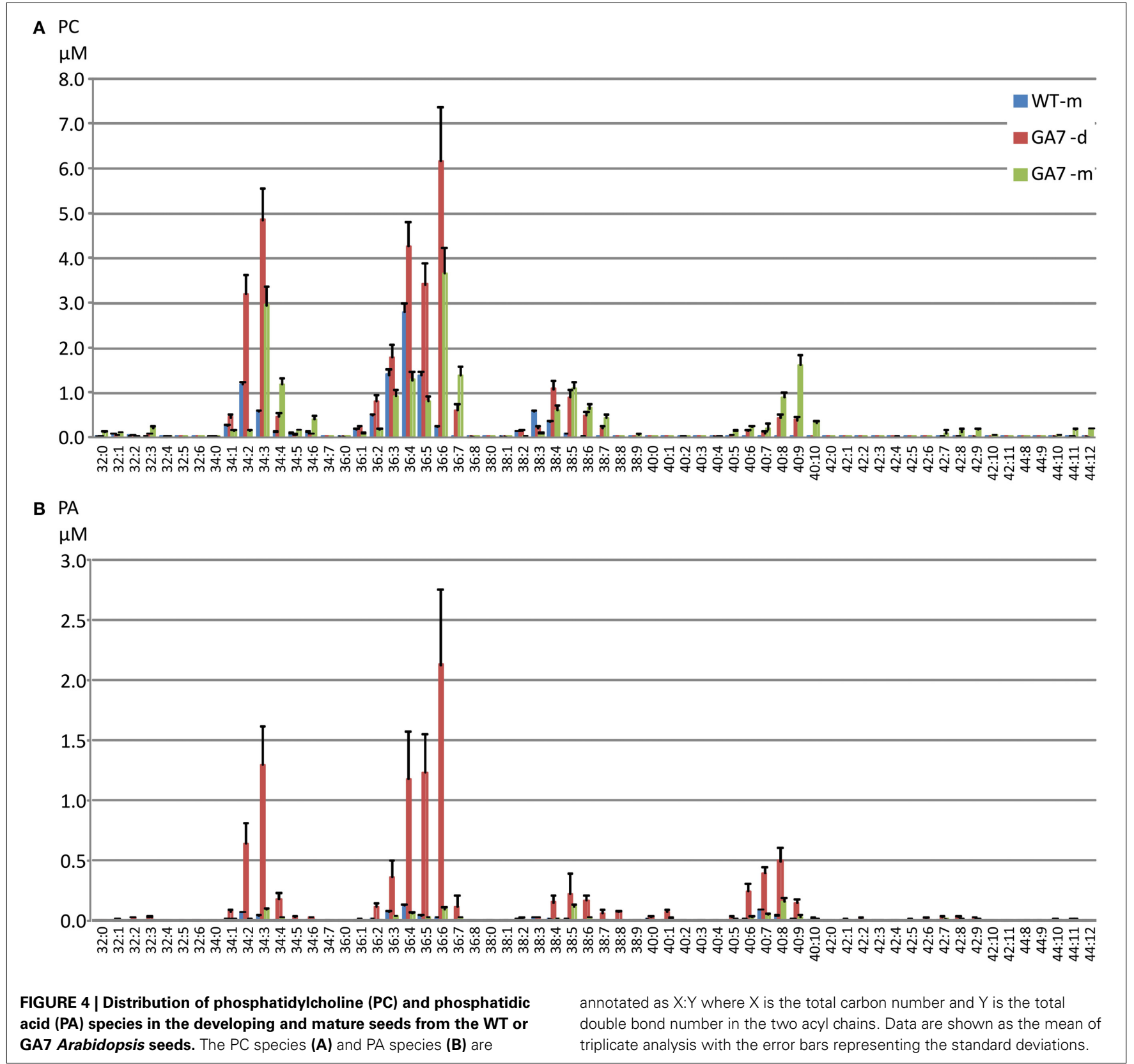


A PE

$\mu \mathrm{M}$

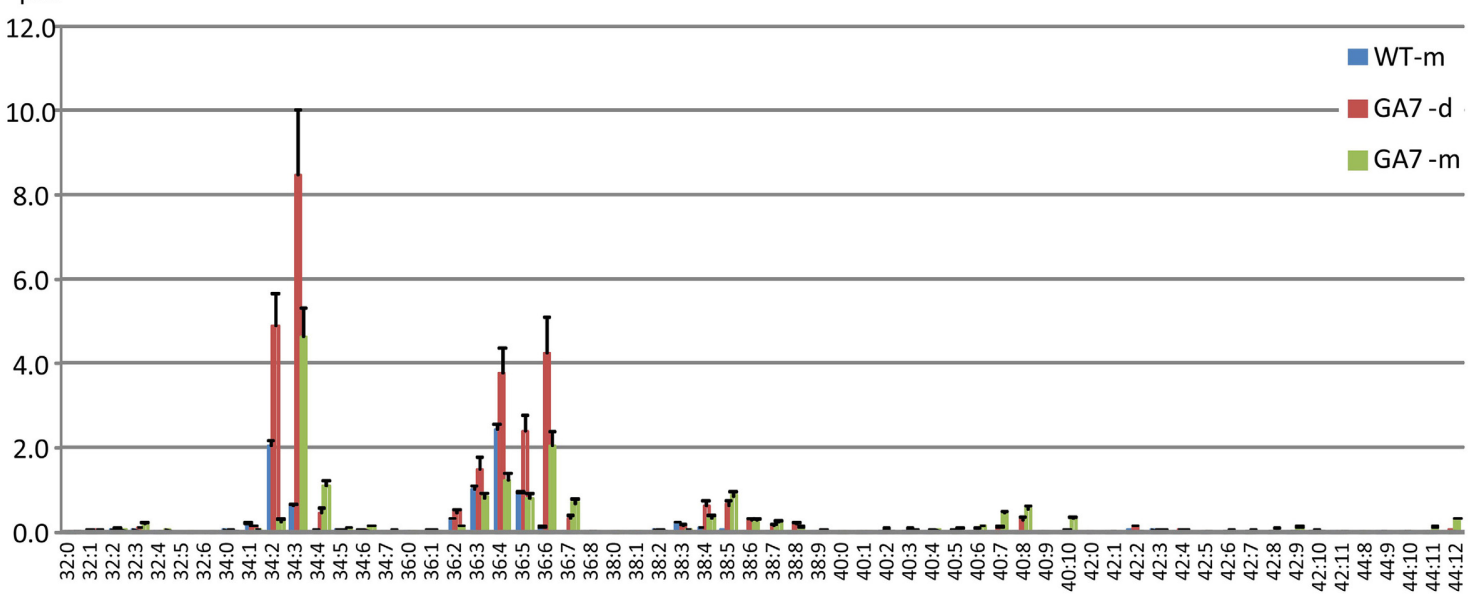

B PG

$\mu \mathrm{M}$
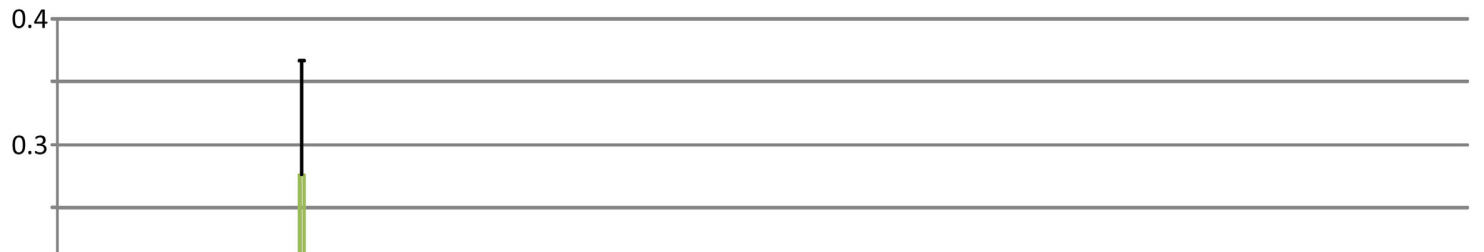

0.2

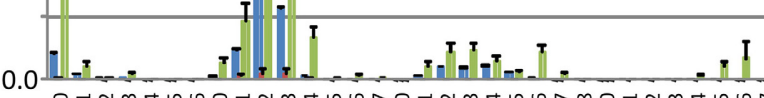

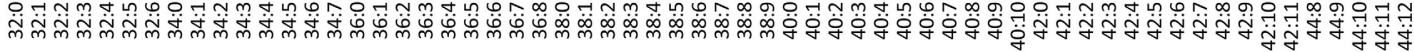

C PI

$\mu \mathrm{M}$

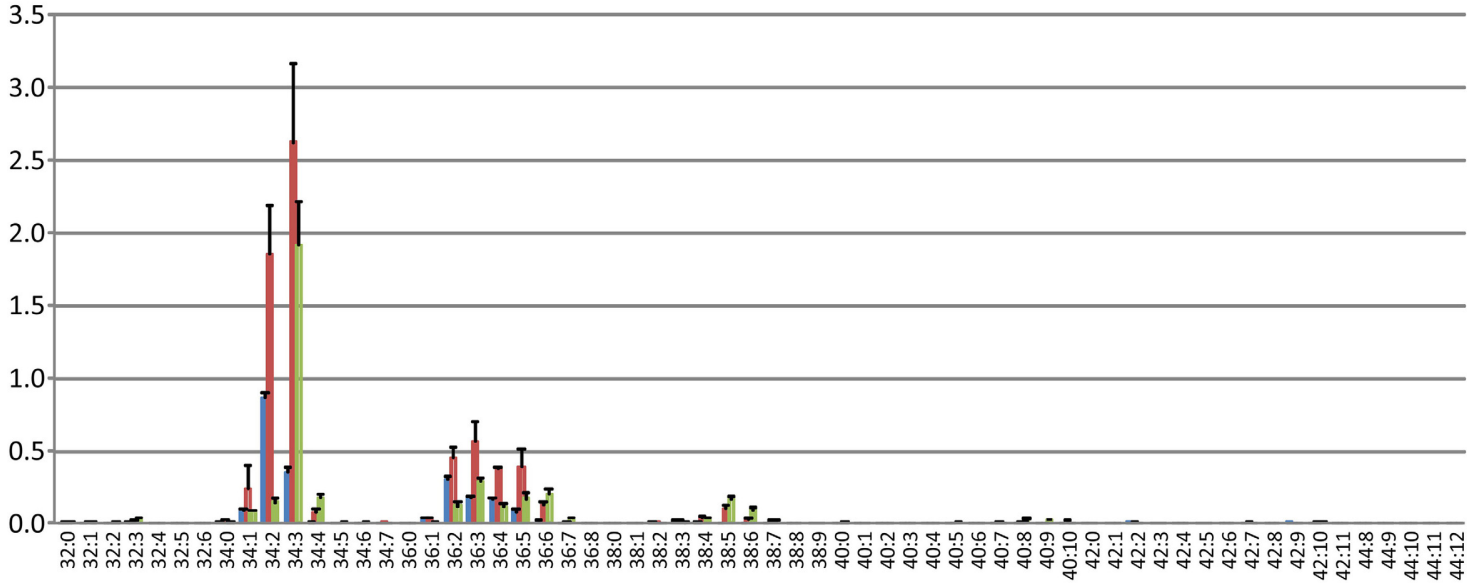

FIGURE 5 | Phosphatidylethanolamine (PE), phosphatidylinositol (PI) and phosphatidylglycerol (PG) pools in the developing and the mature Arabidopsis seeds. The phospholipids PE (A), PG (B) and PI
(C) species are annotated similarly to PC. Data are shown as the mean of triplicate analysis with the error bars representing the standard deviations. 
There was a much lower level of PA in the mature seeds than in the developing seeds based on dry seed weight (Figure 2). It should be noted that lipase activity during extraction could contribute to the higher amount of PA in developing seeds, through conversion of $\mathrm{PC}$ to $\mathrm{PA}$, and therefore a reduction of the $\mathrm{PC}$ concentration would be expected. Two extraction methods were tested using either hot isopropanol quenching (either at 85 or $95^{\circ} \mathrm{C}$ ) or with the procedure described above, and no significant difference in PA concentrations was found by these two methods. Our results showed both PA and PC (and even PE, PI) were higher in developing seeds than in mature seeds (Figure 2), suggesting the higher amount of PA was not an artifact from lipase activity. GA7 had significantly higher amounts of some PA species, especially PA 36:6, PA 36:5, PA 36:4, PA 34:3 and PA 40:8 than other species (Figure 4B). Similar to PC, PA species containing higher numbers of carbons and double bonds in their acyl chains, such as PA 40:6 to PA 40:9, were only found in GA7.

LC-PUFA in other PL groups were also analyzed (Figure 5). PE was the second largest pool of PL. However, only low levels of LC-PUFA appeared in the PE pool. LC-PUFA was much lower in the PI and PG pools. Interestingly, the most significant increase in these three pools of PL in GA7 was 34:3 (possibly composed of palmitic acid and ALA) when compared to the WT. Another interesting aspect was that the much lower levels of PG was found in the GA7 developing seeds, compared to PE and PI. The PS pool was very small, and the LC-MS responses to most of the PS species were close to the detection limit (see Figure S2).

The substantially increased concentration of membrane PL in GA7 seeds when compared to WT seeds, especially the higher levels of PL in developing seeds compared to mature seeds, could be due to the over-expression of the introduced membrane bound fatty acid desaturases and elongases. In higher plants, acyl groups esterified onto PC are the substrates of desaturation by ER membrane desaturases (Sperling et al., 1993). Acyl editing via a PC-deacylation and lysophosphatidylcholine (LPC)-reacylation cycle provided LC-PUFA in the acyl-CoA pool, for further desaturation and elongation by the introduced acyl-CoA dependent enzymes, and for TAG assembly via the Kennedy pathway. The PC-deacylation and LPC-reacylation cycle was catalyzed by acylCoA:LPC acyltransferase (LPCAT) (Bates et al., 2012). Although the engineered pathway mainly targeted acyl-CoA desaturases and elongases, the substantially increased PC pool suggested the importance of the role of PC in accumulation of LC-PUFA in TAG. This suggestion is also supported by Bates and colleagues who showed that the DAG used for TAG synthesis was mostly derived from PC (Bates et al., 2009).

\section{ACCUMULATION OF LC-PUFA CONTAINING DAG AND TAG IN TRANSGENIC SEEDS}

Plants commonly have neutral lipid pools in seeds consisting of $\mathrm{C}_{16}$ to $\mathrm{C}_{22}$ fatty acids with zero to three double bonds. Arabidopsis seeds have low amounts of unsaturated $C_{16}$ fatty acids and $C_{22}$ fatty acids. However, due to the expression of the DHA biosynthesis pathway, there can be more than 20 individual fatty acids in the seed lipids. The total number of theoretical TAG species is 8000 $(20 \times 20 \times 20)$. We therefore targeted only the neutral lipid DAG and TAG species that contain at least one acyl chain consisting of

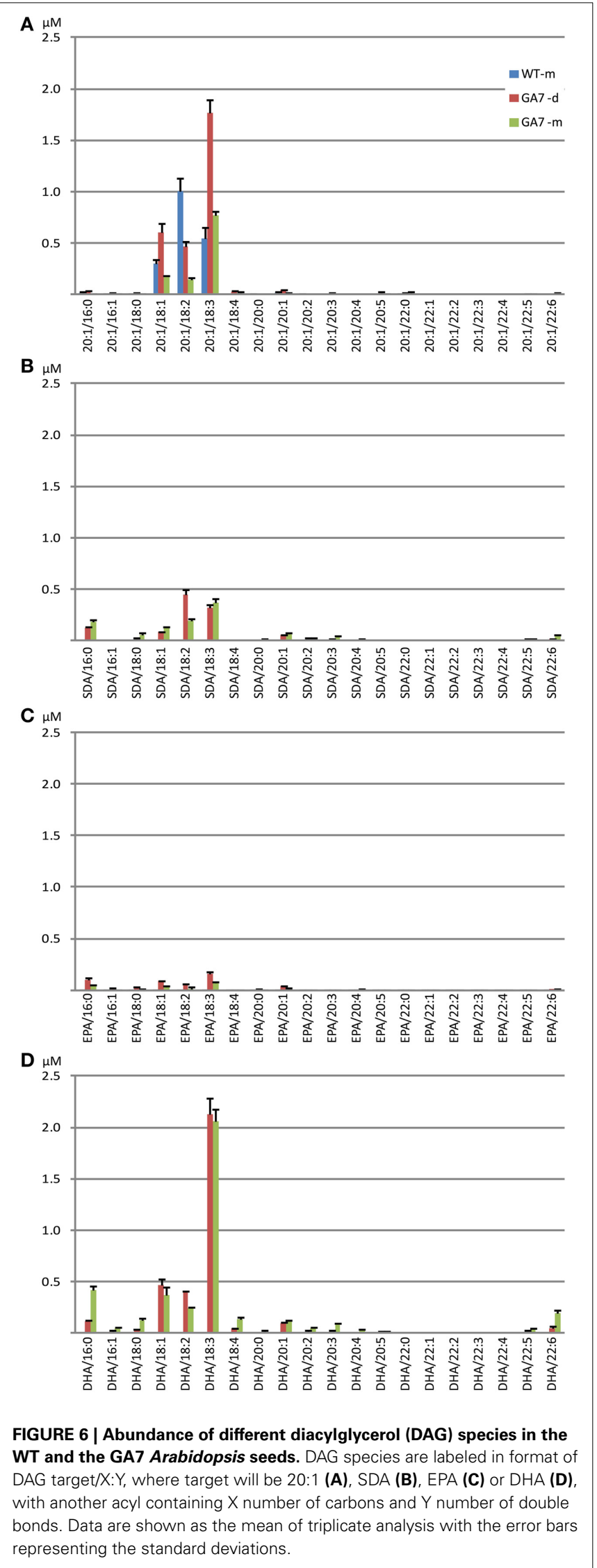


20:1, SDA, EPA, or DHA by setting up MRM's with neutral losses containing one of the four fatty acids of interest. These are shown in the format of TAG 20:1/X:Y, or TAG DHA/X:Y, where X and $\mathrm{Y}$ are the total numbers of carbons and double bonds of fatty acids on the remaining two positions (one position for DAG), respectively.

Analysis of DAG species containing one of the four targeted fatty acids showed that both the GA7 developing and mature seeds accumulated substantial amounts of DHA-containing DAG, with low amounts of DAG containing the intermediate SDA or EPA (Figure S3). In WT, the most abundant 20:1-containing DAG was DAG 20:1/18:2 (52.2\% among all 20:1-containing DAG), followed by DAG 20:1/18:3. In contrast, the GA7 mature seeds showed about five times more DAG 20:1/18:3 than DAG 20:1/18:2 (65.0\% vs. $12.2 \%$ among all 20:1-containing DAG). The major DAG species among all 4 analyzed DAG groups were the DAG molecules containing $\mathrm{C}_{18: 3}$ (Figure 6). In GA7 mature seed lipids, a relatively high percentage of DAG SDA/16:0 and DAG EPA/16:0 was also found, making up $16.0 \%$ of total SDA-containing DAG and $23.0 \%$ of total EPA-containing DAG, although the overall amounts were low (Figures 6B,C). In contrast, DAG DHA/16:0 was found only at $10.7 \%$ of the total DHA-containing DAG, while the predominant DAG DHA/18:3 species was $52.0 \%$.

DHA-containing TAG species were only found in the GA7 developing or mature seeds. The accumulation of LC-PUFAcontaining TAG was at the expense of 20:1-containing TAG (Figure 7). This is in line with previous results reported for total lipids from the GA7 seeds showing that 20:1 was significantly reduced (Petrie et al., 2012). In GA7, most of the 20:1-containing TAG species were reduced when compared to WT, except for the TAG species that also contained ALA or LC-PUFA. For example, TAG 20:1/34:3 (TAG 20:1/16:0/18:3) and TAG 20:1/36:6 (TAG 20:1/18:3/18:3) were increased in GA7 compared to WT (Figure 8A). SDA-, EPA- and DHA-containing TAGs were only found in the GA7 developing or mature seeds. As expected, TAG species 20:1/40:5 to 20:1/40:10, consisting of 20:1 plus LC-PUFA beyond $20: 3$ in the pathway, were absent in WT. This is in contrast to their accumulation, although at low levels in GA7. The highest 20:1-containing TAG species in GA7 were TAG 20:1/36:2 and 20:1/36:6.

TAG species in the GA7 developing and mature seeds containing SDA, EPA or DHA that were absent from WT, are shown in Figures 8B-D. The levels of TAG SDA/34:3, TAG SDA/36:6, TAG SDA/36:7, TAG SDA/38:4 and TAG SDA/40:9 increased significantly from the developing seeds to the mature seeds. There was no significant change in profile of EPA- or DHAcontaining TAG species in the developing or mature seeds. EPA accounted for only $1.8 \%$, compared to $15.1 \%$ of DHA in total seed lipids.

\section{MAJOR NOVEL $\omega 3$ LC-PUFA CONTAINING TAG SPECIES IN THE TRANSGENIC SEEDS}

The most abundant TAG species among the four analyzed groups in mature GA7 seeds were the TAG species that also contained 18:3, i.e., TAG 20:1/36:6, TAG SDA/36:6, TAG EPA/36:6, TAG DHA/36:6. This was followed by TAG 20:1/34:3 and TAG 20:1/36:2, TAG SDA/34:3 and TAG SDA/38:4, TAG EPA/34:3 and TAG EPA/38:4, or TAG DHA/34:3 and TAG DHA/36:5. Very low amounts of tri-DHA TAG was also detected in the GA7 mature seeds. This highlights the utility of LC-MS based lipid profiling as this finding would be impossible with GC-MS based protocols. These identities were confirmed by MS/MS analysis (Figure S4) and summarized in Table 2.The results also suggested a likely preference of LC-PUFA combined with 18:3 in TAG. Based on the TAG fatty acid profile in the mature seed of GA7 which was dominated by $18: 3,22: 6,16: 0$, and 20:1 (Petrie et al., 2012), the TAG species distribution among all the DHA containing TAG species was calculated, if they were randomly associated. The TAG species that were significantly higher in measured association than the randomized prediction mainly contained 18:3 (Table S2). In contrast, the DHA containing TAG species combined with other dominant fatty acids like 16:0 and 20:1 were significantly lower than the randomized prediction. The predicted tri-DHA in all the DHA containing TAG species could be $0.9 \%$, but only $0.1 \%$ was measured. These suggested that DHA distribution in TAG was not randomly. Non-random pattern of elongated acyl chains that are

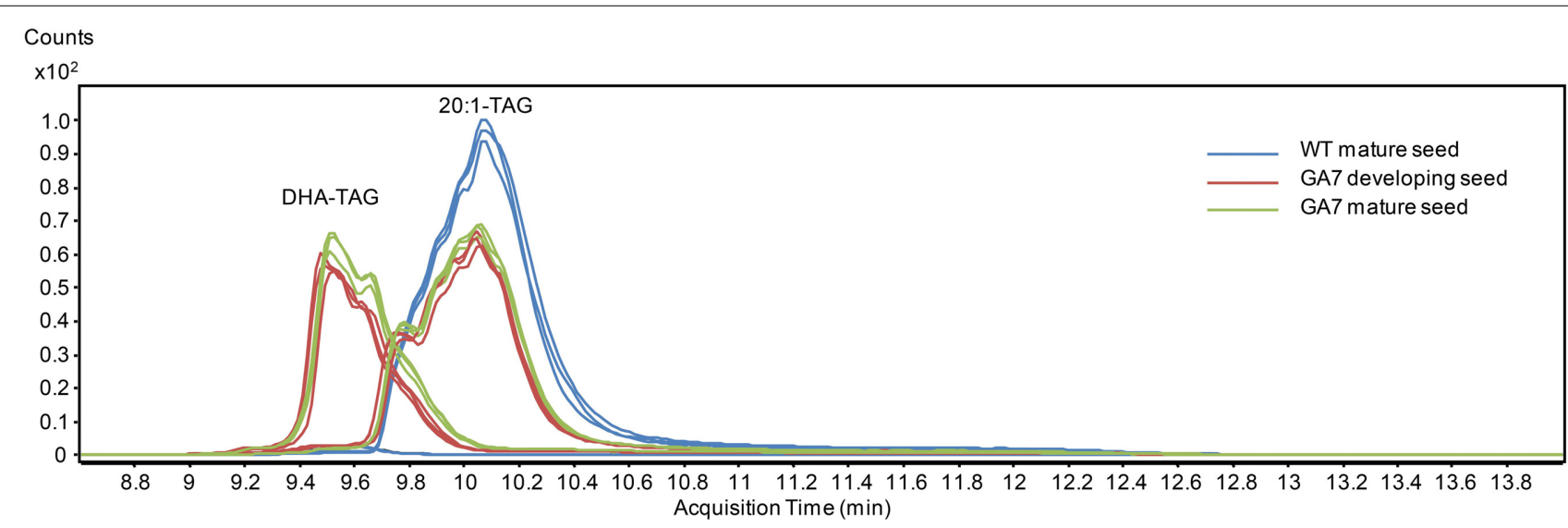

FIGURE 7 | Total ion chromatogram from multiple reaction monitoring (MRM) of the triacylglycerol (TAG) species containing 20:1 or DHA from the WT and the GA7 Arabidopsis seeds. 


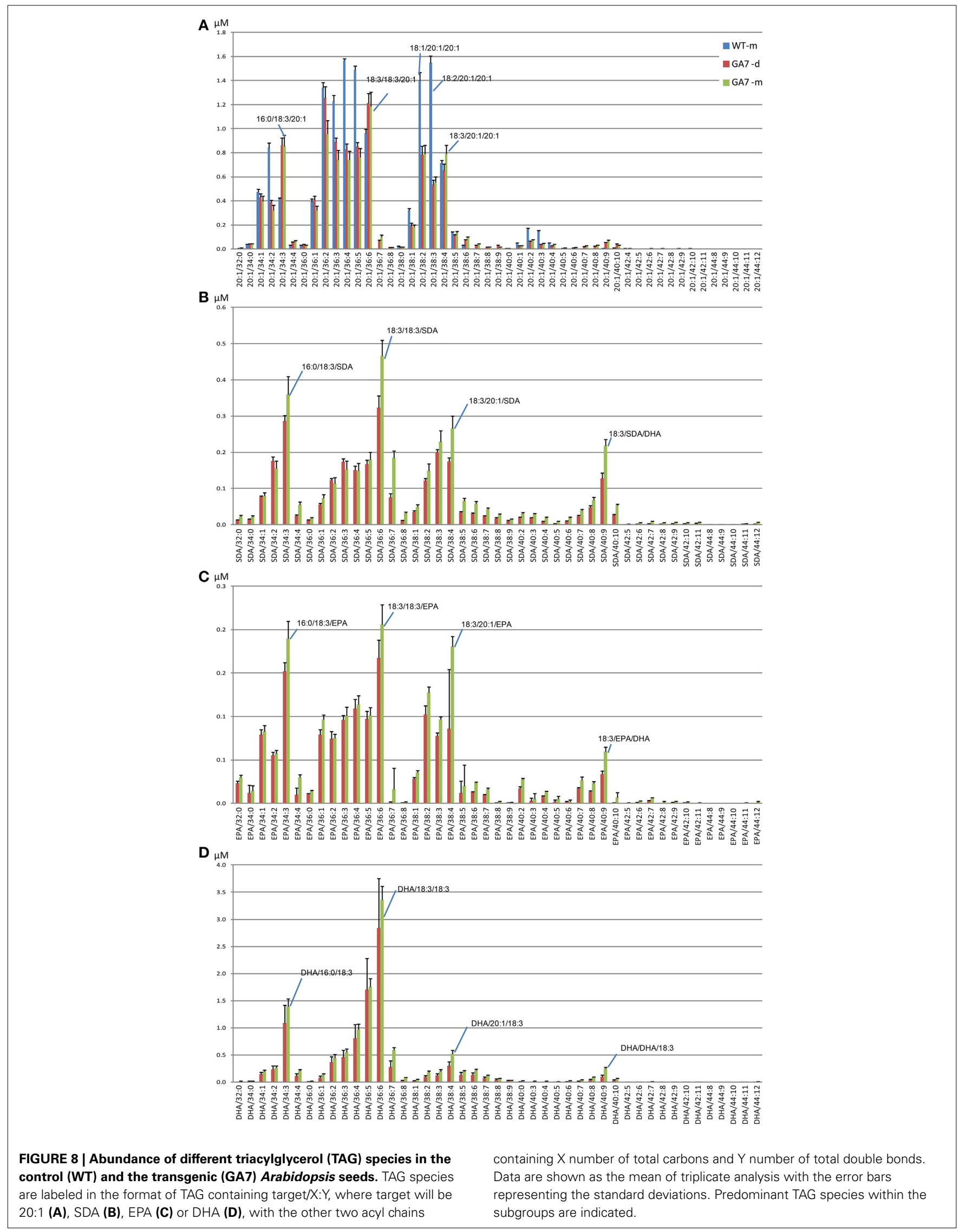


Table 2 | MS/MS fragmentation and structure analysis of the selected TAG species.

\begin{tabular}{lccc}
\hline TAG species & Precursor & Neutral loss & $\begin{array}{c}\text { Major or only } \\
\text { composition of TAG }\end{array}$ \\
\hline TAG 20:1/34:3 & 900.77 & $16: 0,18: 3,20: 1$ & $16: 0 / 18: 3 / 20: 1$ \\
TAG 20:1/36:6 & 922.75 & $18: 3,20: 1$ & $18: 3 / 18: 3 / 20: 1$ \\
TAG 20:1/38:4 & 954.81 & $18: 3,20: 1$ & $18: 3 / 20: 1 / 20: 1$ \\
TAG SDA/34:3 & 866.72 & $16: 0,18: 3$, SDA & $16: 0 / 18: 3 /$ SDA \\
TAG SDA/36:6 & 888.70 & $18: 3$, SDA & $18: 3 / 18: 3 /$ SDA \\
TAG SDA/38:4 & 920.77 & $18: 3$, SDA, 20:1 & $18: 3 /$ SDA/20:1 \\
TAG SDA/40:9 & 938.72 & $18: 3$, SDA, DHA & $18: 3 /$ DA/DHA \\
TAG EPA/34:3 & 892.74 & $16: 0,18: 3$, EPA & $16: 0 / 18: 3 / E P A$ \\
TAG EPA/36:6 & 914.72 & $18: 3$, EPA & $18: 3 / 18: 3 / E P A$ \\
TAG EPA/38:4 & 946.78 & $18: 3,20: 1$, EPA & $18: 3 / 20: 1 / E P A$ \\
TAG EPA/40:9 & 964.74 & $18: 3$, EPA, DHA & $18: 3 / E P A / D H A$ \\
TAG DHA/36:6 & 940.71 & $18: 3$, DHA & $18: 3 / 18: 3 / D H A$ \\
TAG DHA/34:3 & 918.72 & $16: 0,18: 3$, DHA & $16: 0 / 18: 3 / D H A$ \\
TAG DHA/38:4 & 972.77 & $18: 3,18: 4,20: 0$, & $18: 3 / 20: 1 / \mathrm{DHA}$, \\
& & $20: 1$, DHA & $18: 4 / 20: 0 / D H A$ \\
TAG DHA/40:9 & 990.72 & $18: 3$, DHA, DHA & $18: 3 / \mathrm{DHA} / \mathrm{DHA}$ \\
TAG DHA/44:12 & 1040.60 & DHA & DHA/DHA/DHA \\
\hline
\end{tabular}

incorporated into TAG in Arabidopsis seed is recently discovered by LC-MS (Li et al., 2014).

The positional distribution of the three fatty acids in the abundant TAG species could not be resolved by LC-MS analysis. Other studies using NMR analysis of DHA in Arabidopsis seed oil have shown that DHA is preferentially positioned at $s n-1 / 3$ (Petrie et al., 2012). The most abundant DHA-containing DAG or TAG were DAG DHA/18:3 or TAG DHA/18:3/18:3, implying the 2 nd 18:3 fatty acid might be at the $s n-3$ position, thus the

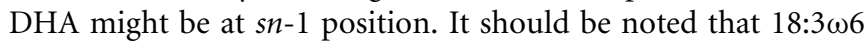
(GLA) and 18:3 $\omega 3$ (ALA) could not be distinguished by LC-MS analysis, GA7 had very low levels of GLA in total seed lipids compared to the high levels of ALA. Therefore, the abundant C18:3 in the DHA-containing TAG might be ALA. The observed low amount of di-DHA PC, di-DHA DAG and tri-DHA TAG also suggested that the transgenic GA7 was able to incorporated DHA onto all three positions of TAG albeit at low levels. A similar observations showing low amounts of tri-EPA TAG (1.5\% of total EPA-containing TAG) was also reported from the transgenic Camelina sativa seed expressing the EPA synthesis pathway (RuizLopez et al., 2013a). DHA esterified onto $s n-1$ position would most likely be contributed by GPAT enzyme, which catalyzes the acylation of glycerol-3-phosphate with acyl-CoA. Although the acyl-CoA pool was not characterized in this study, incorporation of $15 \%$ of DHA in GA7 TAG together with the fact that tri-DHA TAG was also detected, suggested that the appearance of LC-PUFA in acyl-CoA pool did occur. Furthermore, the final step of acylation from DAG to TAG can occur by a number of distinct TAG assembly pathways, including acyl-CoA dependent DGAT, or by the transferring of the acyl group from $s n-2$ of PC to $s n-3$ of DAG by phospholipid:diacylglycerol acyltransferase (PDAT) (Dahlqvist et al., 2000). LC-MS analysis described here was not able to indicate which one of these routes was the major contributor to the TAG assembly. However, the endogenous Arabidopsis AtDAGT2 was shown to prefer to PUFA for the DAG to TAG conversion (Zhou et al., 2013). The AtDGAT2 might favor the assembly of more 18:3 onto DHA containingDAG, resulting in the high proportion of TAG DHA/18:3/18:3 observed in our study.

\section{DHA FROM SYNTHESIS TO STORAGE}

In the mature seeds of GA7, $11.7 \%$ DHA was accumulated in total PC (Table 1). In the total seed lipids of GA7, DHA levels increased to $15.1 \%$. GA7 had the substantial increase of PC 34:3 (PC 16:0/18:3) and PC 36:6 (PC 18:3/18:3), along with significant amounts of TAG DHA/18:3/18:3 and TAG DHA/16:0/18:3. These observations suggest efficient flux from PC 16:0/18:3 and PC 18:3/18:3 to the TAG pool. Indeed, analysis of 18:3-containing DAG species showed DAG 18:3/16:0 and DAG 18:3/18:3 had a significant increase in GA7 when compared to WT (data not shown). This observation was in agreement with that previously proposed for the PC-derived DAG contributing to synthesis of PUFA-containing TAG (Bates and Browse, 2012).

Recently, Ruiz-Lopez et al. (2013a) reported a maximum transgenic production of $14 \%$ DHA in C. sativa. By transgenically expressing a different set of seven enzymes, they found the DHA accumulation in PC was higher than DAG, with even lower levels in TAG, indicating the existence of an inefficient flux of DHA into TAG. This might reflect the different set of enzymes used in these two studies, or due to the different host background. In summary, the accumulation of high levels of DHA in transgenic Arabidopsis seed oil was accompanied with enhanced levels of PL especially PC, DHA-containing DAG and TAG species, as well as the decreased levels of 20:1-containing DAG and TAG. The work also showed evidence that there is a preference of engineered LCPUFA, especially DHA, in neutral and phospholipid containing 18:3. This study demonstrates that LC-MS analysis of lipidome is a invaluable tool for gaining insights into how the engineered fatty acid combine with other fatty acids in lipids of transgenic seeds.

\section{AUTHOR CONTRIBUTIONS}

Conceived and designed the experiment: Xue-Rong Zhou, Surinder P. Singh. Performed the experiment: Xue-Rong Zhou, Damien L. Callahan, Pushkar Shrestha, Qing Liu, James R. Petrie. Analyzed the data: Xue-Rong Zhou, Damien L. Callahan, Pushkar Shrestha, Surinder P. Singh. Contributed reagents/materials/analysis tools: Damien L. Callahan, Pushkar Shrestha. Wrote the paper: Xue-Rong Zhou, Surinder P. Singh. All authors revised the draft and approved the final manuscript.

\section{ACKNOWLEDGMENTS}

This work was supported in part by Bioplatform Australia Services, CSIRO Newton Turner Award, and the CSIRO Food Futures National Research Flagship, CSIRO Plant Industry. LCMS was performed at Metabolomic Australia, University of Melbourne. We thank Lina Ma, Lijun Tian, Bei Dong, Cheryl Blundell, Melanie J. Shears for their excellent technical assistance. We acknowledge Drs. Peter Nichols and Peter Mansour at CSIRO Marine and Atmospheric Research for reviewing the manuscript and providing useful suggestions. 


\section{SUPPLEMENTARY MATERIAL}

The Supplementary Material for this article can be found online at: http://www.frontiersin.org/journal/10.3389/fpls.2014. 00419/abstract

Figure S1 | MS/MS analysis of phosphotidylcholine (PC) precursor at $\mathrm{m} / \mathrm{z}$ 878.6 with a PC head group ion of $\mathbf{m} / \mathbf{z}$ 184.2. $Y$ axis represents the response of ion scan. The low abundant fragment at $\mathrm{m} / \mathrm{z} 568.3$ is due to the neutral loss of 22:6, thus confirming the identity as PC 22:6/22:6.

Figure S2 | Low abundance phosphatidylserine (PS) pool in the developing and the mature Arabidopsis seeds. The PS are annotated similarly to PC. Data are shown as the mean of triplicate analysis with the error bars representing the standard deviations.

Figure S3 | The abundances of long-chain polyunsaturated fatty acids (LC-PUFA) containing diacylglecerol (DAG) in the WT and GA7 Arabidopsis

seeds. Data are shown as the mean of triplicate analysis with the error bars representing the standard deviations.

Figure S4 | Product ion spectra for identification of selected lipid species. (A) Fragmentation of the $\left[\mathrm{M}+\mathrm{NH}_{4}^{+}\right]^{+}$ion at $940.75 \mathrm{~m} / \mathrm{z}$ showing the neutral losses of $18: 3$ and 22:6, represents a triacylglycerol (TAG) composed of 18:3/18:3/DHA. (B) Fragmentation of the $\left[\mathrm{M}+\mathrm{NH}_{4}\right]^{+}$ion at $922.79 \mathrm{~m} / \mathrm{z}$ showing the neutral losses of $18: 3$ and $20: 1$, represents a TAG composed of 18:3/18:3/20:1. (C) Fragmentation of the $\left[\mathrm{M}+\mathrm{NH}_{4}\right]^{+}$ion $1040.59 \mathrm{~m} / \mathrm{z}$ showing the neutral loss of $22: 6$, represents a TAG composed with tri-DHA.

\section{REFERENCES}

Bates, P. D., and Browse, J. (2012). The significance of different diacylgycerol synthesis pathways on plant oil composition and bioengineering. Front. Plant Sci. 3:147. doi: $10.3389 /$ fpls.2012.00147

Bates, P. D., Durrett, T. P., Ohlrogge, J. B., and Pollard, M. (2009). Analysis of Acyl Fluxes through multiple pathways of Triacylglycerol synthesis in developing Soybean embryos. Plant Physiol. 150, 55-72. doi: 10.1104/pp.109. 137737

Bates, P. D., Fatihi, A., Snapp, A. R., Carlsson, A. S., Browse, J., and Lu, C. (2012). Acyl editing and headgroup exchange are the major mechanisms that direct polyunsaturated fatty acid flux into triacylglycerols. Plant Physiol. 160, 1530-1539. doi: 10.1104/pp.112.204438

Bates, P. D., Johnson, S. R., Cao, X., Li, J., Nam, J. W., Jaworski, J. G., et al. (2014). Fatty acid synthesis is inhibited by inefficient utilization of unusual fatty acids for glycerolipid assembly. Proc. Natl. Acad. Sci. U.S.A. 111, 1204-1209. doi: 10.1073/pnas.1318511111

Blanksby, S. J., and Mitchell, T. W. (2010). Advances in mass spectrometry for lipidomics. Annu. Rev. Anal. Chem. 3, 433-465. doi: 10.1146/annurev.anchem. 111808.073705

Dauk, M., Lam, P., Kunst, L., and Smith, M. A. (2007). A FAD2 homologue from Lesquerella lindheimeri has predominantly fatty acid hydroxylase activity. Plant Sci. 173, 43-49. doi: 10.1016/j.plantsci.2007.03.015

Dahlqvist, A., Stahl, U., Lenman, M., Banas, A., Lee, M., Sandager, L., et al. (2000). Phospholipid:diacylglycerol acyltransferase: an enzyme that catalyzes the acylCoA-independent formation of triacylglycerol in yeast and plants. Proc. Natl. Acad. Sci. U.S.A. 97, 6487-6492. doi: 10.1073/pnas.120067297

Ferreri, C., and Chatgilialoglu, C. (2012). Role of fatty acid-based functional lipidomics in the development of molecular diagnostic tools. Expert Rev. Mol. Diagn. 12, 767-780. doi: 10.1586/erm.12.73

Han, X., and Gross, R. W. (2005). Shotgun lipidomics: multidimensional MS analysis of cellular lipidomes. Expert Rev. Proteomics 2, 253-264. doi: 10.1586/ 14789450.2.2.253

Haslam, R. P., Ruiz-Lopez, N., Eastmond, P., Moloney, M., Sayanova, O., and Napier, J. A. (2013). The modification of plant oil composition via metabolic engineering-better nutrition by design. Plant Biotechnol. J. 11, 157-168. doi: $10.1111 /$ pbi.12012
$\mathrm{Hu}, \mathrm{Z}$., Ren, Z., and Lu, C. (2012). The phosphatidylcholine diacylglycerol cholinephosphotransferase is required for efficient hydroxy fatty acid accumulation in transgenic Arabidopsis. Plant physiol. 158, 1944-1954. doi: 10.1104/pp. 111.192153

Hyötyläinen, T., Bondia-Pons, I., and Orešiè, M. (2013). Lipidomics in nutrition and food research. Mol. Nutr. Food Res. 57, 1306-1318. doi: 10.1002/mnfr. 201200759

James, M. J., Gibson, R. A., and Cleland, L. G. (2000). Dietary polyunsaturated fatty acids and inflammatory mediator production. Am. J. Clin. Nutr. 71, 343s-348s.

Khozin, I., Adlerstein, D., Bigongo, C., Heimer, Y. M., and Cohen, Z. (1997). Elucidation of the biosynthesis of eicosapentaenoic acid in the microalga Porphyridium cruentum.2. Studies with radiolabeled precursors. Plant Physiol. 114, 223-230.

Li, M., Baughman, E., Roth, M. R., Han, X., Welti, R., and Wang, X. (2014). Quantitative profiling and pattern analysis of triacylglycerol species in Arabidopsis seeds by electrospray ionization mass spectrometry. Plant J. 77, 160-172. doi: 10.1111/tpj.12365

Li, R. Z., Yu, K. S., Wu, Y. M., Tateno, M., Hatanaka, T., and Hildebrand, D. F. (2012). Vernonia DGATs can complement the disrupted oil and protein metabolism in epoxygenase-expressing soybean seeds. Metab. Eng. 14, 29-38. doi: 10.1016/j.ymben.2011.11.004

Maatta, S., Scheu, B., Roth, M. R., Tamura, P., Li, M. Y., Williams, T. D., et al. (2012). Levels of Arabidopsis thaliana leaf phosphatidic acids, phosphatidylserines, and most trienoate-containing polar lipid molecular species increase during the dark period of the diurnal cycle. Front. Plant Sci. 3:49. doi: 10.3389/fpls.2012.00049

Petrie, J. R., Shrestha, P., Zhou, X.-R., Mansour, M. P., Liu, Q., Belide, S., et al. (2012). Metabolic engineering plant seeds with fish oil-like levels of DHA. PLoS ONE 7:e49165. doi: 10.1371/journal.pone.0049165

Robert, S. S., Singh, S. P., Zhou, X.-R., Petrie, J. R., Blackburn, S. I., Mansour, P. M., et al. (2005). Metabolic engineering of Arabidopsis to produce nutritionally important DHA in seed oil. Funct. Plant Biol. 32, 473-479. doi: 10.1071/ FP05084

Ruiz-Lopez, N., Haslam, R. P., Napier, J. A., and Sayanova, O. (2013a). Successful high-level accumulation of fish oil omega-3 long-chain polyunsaturated fatty acids in a transgenic oilseed crop. Plant J. 18, 198-208. doi: 10.1111/tpj. 12378

Ruiz-Lopez, N., Haslam, R. P., Usher, S. L., Napier, J. A., and Sayanova, O. (2013b). Reconstitution of EPA and DHA biosynthesis in Arabidopsis: Iterative metabolic engineering for the synthesis of n-3 LC-PUFAs in transgenic plants. Metab. Eng. 17, 30-41. doi: 10.1016/j.ymben.2013. 03.001

Ruiz-Lopez, N., Sayanova, O., Napier, J. A., and Haslam, R. P. (2012). Metabolic engineering of the omega-3 long chain polyunsaturated fatty acid biosynthetic pathway into transgenic plants. J. Exp. Bot. 63, 2397-2410. doi: 10.1093/jxb/ err454

Slack, C. R., Campbell, L. C., Browse, J. A., and Roughan, P. G. (1983). Some evidence for the reversibility of the cholinephosphotransferase-catalysed reaction in developing linseed cotyledons in vivo. Biochim. Biophys. Acta 754, 10-20. doi: 10.1016/0005-2760(83)90076-0

Sperling, P., Linscheid, M., Stocker, S., Muhlbach, H. P., and Heinz, E. (1993). In vivo desaturation of cis-delta 9-monounsaturated to cisdelta 9,12-diunsaturated alkenylether glycerolipids. J. Biol. Chem. 268, 26935-26940.

Stymne, S., and Stobart, A. K. (1984). Evidence for the reversibility of the acyl-CoA:lysophosphatidylcholine acyltransferase in microsomal preparations from developing safflower (Carthamus tinctorius L.) cotyledons and rat liver. Biochem. J. 223, 305-314.

Wang, L., Shen, W., Kazachkov, M., Chen, G., Chen, Q., Carlsson, A. S., et al. (2012). Metabolic interactions between the Lands cycle and the Kennedy pathway of glycerolipid synthesis in Arabidopsis developing seeds. Plant Cell 24, 4652-4669. doi: 10.1105/tpc.112.104604

Watson, A. D. (2006). Thematic review series: Systems biology approaches to metabolic and cardiovascular disorders. Lipidomics: a global approach to lipid analysis in biological systems. J. Lipid Res. 47, 2101-2111. doi: 10.1194/jlr. R600022-JLR200

Williams, J. P., Imperial, V., Khan, M. U., and Hodson, J. N. (2000). The role of phosphatidylcholine in fatty acid exchange and desaturation in 
Brassica napus L. leaves. Biochem. J. 349, 127-133. doi: 10.1042/0264-6021: 3490127

Wu, G. H., Truksa, M., Datla, N., Vrinten, P., Bauer, J., Zank, T., et al. (2005). Stepwise engineering to produce high yields of very long-chain polyunsaturated fatty acids in plants. Nat. Biotechnol. 23, 1013-1017. doi: 10.1038/ nbt1107

Zhou, X.-R., Shrestha, P., Yin, F., Petrie, J. R., and Singh, S. P. (2013). AtDGAT2 is a functional acyl-CoA:diacylglycerol acyltransferase and displays different acyl-CoA substrate preferences than AtDGAT1. Febs Lett. 587, 2371-2376. doi: 10.1016/j.febslet.2013.06.003

Conflict of Interest Statement: The authors declare that the research was conducted in the absence of any commercial or financial relationships that could be construed as a potential conflict of interest.
Received: 08 May 2014; accepted: 05 August 2014; published online: 01 September 2014.

Citation: Zhou X-R, Callahan DL, Shrestha P, Liu Q, Petrie JR and Singh SP (2014) Lipidomic analysis of Arabidopsis seed genetically engineered to contain DHA. Front. Plant Sci. 5:419. doi: 10.3389/fpls.2014.00419

This article was submitted to Plant Metabolism and Chemodiversity, a section of the journal Frontiers in Plant Science.

Copyright (c) 2014 Zhou, Callahan, Shrestha, Liu, Petrie and Singh. This is an open-access article distributed under the terms of the Creative Commons Attribution License (CC BY). The use, distribution or reproduction in other forums is permitted, provided the original author(s) or licensor are credited and that the original publication in this journal is cited, in accordance with accepted academic practice. No use, distribution or reproduction is permitted which does not comply with these terms. 